\title{
Unraveling the Large Deviation Statistics of Markovian Open Quantum Systems
}

\author{
Federico Carollo, ${ }^{1}$ Robert L. Jack, ${ }^{2}$ and Juan P. Garrahan ${ }^{1}$ \\ ${ }^{1}$ School of Physics and Astronomy and Centre for the Mathematics and Theoretical Physics of Quantum Non-Equilibrium Systems, \\ University of Nottingham, Nottingham, NG7 2RD, United Kingdom \\ ${ }^{2}$ Department of Applied Mathematics and Theoretical Physics, University of Cambridge, \\ Wilberforce Road, Cambridge CB3 OWA, United Kingdom \\ and Department of Chemistry, University of Cambridge, Lensfield Road, Cambridge CB2 1EW, United Kingdom
}

(Received 3 December 2018; revised manuscript received 5 March 2019; published 5 April 2019)

\begin{abstract}
We analyze dynamical large deviations of quantum trajectories in Markovian open quantum systems in their full generality. We derive a quantum level-2.5 large deviation principle for these systems, which describes the joint fluctuations of time-averaged quantum jump rates and of the time-averaged quantum state for long times. Like its level-2.5 counterpart for classical continuous-time Markov chains (which it contains as a special case), this description is both explicit and complete, as the statistics of arbitrary timeextensive dynamical observables can be obtained by contraction from the explicit level-2.5 rate functional we derive. Our approach uses an unraveled representation of the quantum dynamics which allows these statistics to be obtained by analyzing a classical stochastic process in the space of pure states. For quantum reset processes we show that the unraveled dynamics is semi-Markovian and derive bounds on the asymptotic variance of the number of quantum jumps which generalize classical thermodynamic uncertainty relations. We finish by discussing how our level-2.5 approach can be used to study large deviations of nonlinear functions of the state, such as measures of entanglement.
\end{abstract}

DOI: 10.1103/PhysRevLett.122.130605

Introduction.-Practical quantum systems are always coupled to their environments, which means that their dynamics are stochastic. This is manifested, e.g., by wave function collapse and by decoherence. In such open quantum systems one aims to trace out the environment and follow the dynamics of the system state [1-4]. In many situations, this leads to a density matrix $\rho$ that evolves deterministically in continuous time, according to a Markovian quantum master equation (QME). This dynamics can be understood via a mapping to stochastic quantum trajectories [5-8] — this is called unraveling the QME. An individual quantum trajectory specifies the behavior of the system conditioned on a time record of observations (or events) in the environment. If the events are quantum jumps (as, e.g., in the case of photon counting), the trajectories are those of a continuous-time quantum Markov chain; see Refs. [1-4]. Averaging over these recovers the QME, but information about their fluctuations requires knowledge about the quantum trajectories.

The state-of-the art approach for characterizing fluctuations in stochastic trajectories uses large deviation principles (LDPs) [9-17]. This method focuses on rare events in which time-averaged quantities deviate significantly from their typical (ergodic) values. In open quantum systems, LDPs have been used to analyze the counting statistics of quantum jumps [18-24] and of homodyne currents [25]. For classical systems, two important recent advances have been the analysis of LDPs for the full statistics of all fluxes and state occupancies (LDPs at level 2.5 [26-31]) and variational analyses based on optimal control theory $[32,33]$. Here, we extend these ideas to stochastic quantum trajectories.

In particular, we establish a level-2.5 LDP for quantum jump trajectories, including variational representations of rate functions, based on optimal-control theory. This framework recovers previous results for the statistics of arbitrary dynamical observables (by using a contraction principle [13]). In addition, it enables several new applications, two of which we consider in detail. First, the level2.5 LDP allows us to derive bounds on the precision of estimation of the (empirical) rates of quantum jumps in quantum reset processes, thus generalizing classical thermodynamic uncertainty relations [34-38]. Second, the level-2.5 LDP can be used to analyze new kinds of dynamical fluctuations, which are related to nonlinear functions of the state; as an example, we consider fluctuations of the bipartite entanglement entropy.

Average and unraveled dynamics.-We consider Markovian open quantum systems in continuous time, where the system density matrix $\rho_{t}$ evolves according to a QME, $\partial \rho_{t} / \partial t=\mathcal{L}\left(\rho_{t}\right)$. The Lindbladian $\mathcal{L}[39,40]$ acts on density matrices as [1-3]

$$
\mathcal{L}(\cdot)=-i[H,(\cdot)]+\sum_{i}\left(J_{i}(\cdot) J_{i}^{\dagger}-\frac{1}{2}\left\{J_{i}^{\dagger} J_{i},(\cdot)\right\}\right)
$$


where $J_{i}$ is a jump operator, and $i=1,2, \ldots, m$ identifies the type of quantum jump. For example, different types of jumps might correspond to emitted photons with different frequencies. We write $[A, B]=A B-B A$ for the commutator of two operators and $\{A, B\}=A B+B A$ for their anticommutator.

Our approach is based on unraveling the dynamics described by the QME in terms of quantum jump trajectories [1-3]. Each trajectory consists of the stochastic evolution of a pure state, which we denote at time $t$ by the rank-1 density matrix $\psi_{t}$, conditioned on a given time record of jump events. The pure state $\psi_{t}$ thus evolves according to the Belavkin stochastic differential equation (SDE) [5],

$$
d \psi_{t}=\mathcal{B}\left(\psi_{t}\right) d t+\sum_{i}\left(\frac{\mathcal{J}_{i}\left(\psi_{t}\right)}{\operatorname{Tr}\left[\mathcal{J}_{i}\left(\psi_{t}\right)\right]}-\psi_{t}\right) d n_{i t},
$$

where

$$
\mathcal{B}(\psi)=-i H_{\mathrm{eff}} \psi+i \psi H_{\mathrm{eff}}^{\dagger}-\psi \operatorname{Tr}\left(-i H_{\mathrm{eff}} \psi+i \psi H_{\mathrm{eff}}^{\dagger}\right),
$$

with $H_{\text {eff }}=H-(i / 2) \sum_{i} J_{i}^{\dagger} J_{i}$ being the (non-Hermitian) effective Hamiltonian, and $\mathcal{J}_{i}(\psi)=J_{i} \psi J_{i}^{\dagger}$. Formally, the "noise increment" $d n_{i t}$ is equal to one if a jump of type $i$ takes place between times $t$ and $t+d t$ or zero otherwise. The average of $d n_{i t}$ is $\operatorname{Tr}\left[\mathcal{J}_{i}\left(\psi_{t}\right)\right] d t$, and the noise increments obey "Ito rules" $d n_{i t} d n_{j t}=\delta_{i j} d n_{i t}$ [2,3]. Two standard results are [6] (i) the Belavkin SDE maintains $\psi_{t}$ as a pure state, and (ii) for appropriate initial conditions on $\psi_{t}$, the density matrix can be recovered by averaging $\psi$ over the noise realizations: $\rho_{t}=\mathbb{E}\left[\psi_{t}\right]$. Hence, all quantum observables can be computed as classical expectation values for the unraveled process.

Quantum-classical correspondence and master equation for unraveled dynamics.-Equation (2) represents the quantum Markov chain via a classical SDE in the space of pure states $\psi$. Let $P_{t}(\psi)$ be the probability density for $\psi$ at time $t$, in analogy with classical stochastic processes. Then

$$
\begin{aligned}
\partial_{t} P_{t}(\psi)= & -\operatorname{div}\left[\mathcal{B}(\psi) P_{t}(\psi)\right] \\
& +\sum_{i} \int d \psi^{\prime}\left[P_{t}\left(\psi^{\prime}\right) w_{i}\left(\psi^{\prime}, \psi\right)-P_{t}(\psi) w_{i}\left(\psi, \psi^{\prime}\right)\right],
\end{aligned}
$$

where

$$
w_{i}\left(\psi, \psi^{\prime}\right)=\operatorname{Tr}\left[\mathcal{J}_{i}(\psi)\right] \delta\left(\psi^{\prime}-\frac{\mathcal{J}_{i}(\psi)}{\operatorname{Tr}\left[\mathcal{J}_{i}(\psi)\right]}\right)
$$

is the rate for transitions from $\psi$ to $\psi^{\prime}$ due to quantum jump $i$. Precise definitions of the quantities in Eq. (3) are given in
Ref. [41]. We call Eq. (3) the unraveled dynamics quantum master equation (UQME).

Physically, Eqs. (2) and (3) have a simple meaning: the pure state $\psi_{t}$ evolves deterministically along paths specified by the operator $\mathcal{B}$, but this deterministic evolution is punctuated at random times by jumps, specified by $\mathcal{J}_{i}$. The probability $P_{t}$ evolves according to the UQME, and at long times it tends to the stationary solution $P_{\infty}(\psi)$. We assume that this solution is unique, which is the case in most physical applications; see also Ref. [42].

We summarize this quantum-classical mapping (or unraveling) as (i) $\psi_{t}$ is the (stochastic) position in Hilbert space which evolves according to the SDE Eq. (2), (ii) the state $\rho_{t}$ corresponds to the average position and evolves according to the QME Eq. (1) [43], and (iii) the master equation for the stochastic process $\psi_{t}$ is the UQME.

Level-2.5 LDP for unraveled dynamics. - We derive a LDP at level 2.5 for the unraveled process by generalizing the classical result to systems that evolve by a combination of continuous deterministic evolution and discrete (random) jumps, cf. Eqs. (2)-(4). The large deviation (LD) theory of stochastic dynamics is concerned with the behavior of observables that are time integrated over trajectories, for some long time $\tau$ [44]. At level 2.5 these observables fall into two main classes [26-30]: empirical fluxes $q_{\tau}^{i}\left(\psi, \psi^{\prime}\right)$, corresponding to the number of jumps from $\psi$ to $\psi^{\prime}$ per unit time in a trajectory (i.e., empirical transition rates), and the empirical measure $\mu_{\tau}(\psi)$, corresponding to the fraction of time that the system spends in $\psi$. Their (steady-state) averages over trajectories are $\mathbb{E}\left[\mu_{\tau}(\psi)\right]=P_{\infty}(\psi)$ and $\mathbb{E}\left[q_{\tau}^{i}\left(\psi, \psi^{\prime}\right)\right]=P_{\infty}(\psi) w_{i}\left(\psi, \psi^{\prime}\right)$. The level-2.5 LDP quantifies the (small) probability that $\mu_{\tau}$ and $q_{\tau}$ differ from their average values: as $\tau \rightarrow \infty$, then

$$
\operatorname{Prob}\left[\left(\mu_{\tau}, q_{\tau}\right) \approx(\mu, q)\right] \simeq \exp \left(-\tau I_{2.5}[\mu, q]\right),
$$

where $I_{2.5}[\mu, q]$ is the level-2.5 rate functional.

To obtain a formula for $I_{2.5}$, we define a controlled stochastic process, in which the transition rates $w_{i}$ are replaced by auxiliary rates $[32,33]$,

$$
w_{i}^{A}\left(\psi, \psi^{\prime}\right)=A_{i}(\psi) w_{i}\left(\psi, \psi^{\prime}\right),
$$

where $A_{i}$ is a (positive) rescaling factor. The steady-state probability density for this controlled process is denoted by $P_{\infty}^{A}(\psi)$, which may (in principle) be obtained as the steadystate solution of a suitable UQME. By considering cumulant generating functions for $\mu, q$ and performing a Legendre transformation (see Ref. [41] for details), we obtain

$$
I_{2.5}[\mu, q]=\inf _{A} \mathcal{I}\left[w^{A} \mid w\right],
$$

where the infimum is taken over all possible choices of the rescaling factors $A$ such that the controlled process realizes 
the rare values of $\mu, q$; that is, $P_{\infty}^{A}(\psi)=\mu(\psi)$ and $P_{\infty}^{A}(\psi) w_{i}^{A}\left(\psi, \psi^{\prime}\right)=q^{i}\left(\psi, \psi^{\prime}\right)$. If there is no choice for $A$ that satisfies this constraint, then $I_{2.5}[\mu, q]=\infty$. The controlled process that corresponds to the minimizer in Eq. (7) is the optimally controlled process. The quantity to be minimized is a relative entropy,

$$
\mathcal{I}\left[w^{A} \mid w\right]=\int d \psi d \psi^{\prime} P_{\infty}^{A}(\psi) \sum_{i} \mathcal{D}\left[w_{i}^{A}\left(\psi, \psi^{\prime}\right) \mid w_{i}\left(\psi, \psi^{\prime}\right)\right],
$$

where

$$
\mathcal{D}(x \mid y)=x \log (x / y)-x+y .
$$

Using the formulas for $w_{i}^{A}$ and the UQME Eq. (3), one has

$$
I_{2.5}[\mu, q]=\int d \psi d \psi^{\prime} \sum_{i} \mathcal{D}\left[q^{i}\left(\psi, \psi^{\prime}\right) \mid \mu(\psi) w_{i}\left(\psi, \psi^{\prime}\right)\right],
$$

which is valid as long as the continuity constraint,

$$
\operatorname{div}[\mathcal{B}(\psi) \mu(\psi)]=\sum_{i} \int d \psi^{\prime}\left[q^{i}\left(\psi^{\prime}, \psi\right)-q^{i}\left(\psi, \psi^{\prime}\right)\right],
$$

is satisfied for all $\psi$. Otherwise, $I_{2.5}=\infty$. Equations (10) and (11) are analogous to the classical theory of LDs at level 2.5, but generalized to quantum Markovian dynamics, in which the system evolves deterministically between its (random) jumps [45]. Equations (5)-(11) show how large deviations for open quantum dynamics can be analyzed at level 2.5, and they establish a variational principle for the rate function. This is the first main result of the Letter. We now discuss its consequences.

Contraction to level-1 LDPs and quantum Doob transform.-As in the classical case, the level-2.5 LDP rate function is given by an explicit expression, in terms of empirical fluxes and the empirical measure, cf. Eqs. (5)(11). This LDP is complete in the sense that the rate function for any linear combination of the empirical fluxes and measure can be derived from Eq. (10) by the contraction principle of large deviation theory. For example, the number of quantum jumps of type $i$ per unit time is the integral of the empirical flux over all possible initial and final states: $Q_{\tau}^{i}=\int d \psi d \psi^{\prime} q_{\tau}^{i}\left(\psi, \psi^{\prime}\right)$. These fluxes obey a LDP (known as "level 1" [13]): $\operatorname{Prob}\left[Q_{\tau} \approx Q\right] \simeq e^{-\tau I_{1}(Q)}$. The rate function $I_{1}$ can be obtained by contraction from level 2.5; that is, $I_{1}(Q)=\min _{\mu, q: Q} I_{2.5}[\mu, q]$, where the minimization is over all $(\mu, q)$ such that the jump rate is $Q$.

A second important result for level-1 statistics that can be recovered from our level-2.5 approach is the quantum Doob transformation $[18,24]$. This states that there is an auxiliary quantum process for which the rare events in $\operatorname{Prob}\left[Q_{\tau} \approx Q\right]$ become typical. The derivation consists of three main steps: first, the variational characterization of $I_{2.5}$ in Eq. (7) provides an auxiliary process on the space of pure states, which optimally realizes the fluctuation $(\mu, q)$; second, applying a similarity transformation to $\psi_{t}$ yields a new set of quantum stochastic trajectories; third, one shows that these trajectories are an unraveled representation of the Doob-transformed dynamics. For details, see Ref. [41].

These results show that the quantum level-2.5 LDP Eq. (5) can be used to recover existing results that are usually calculated through tilted Lindbladian methods $[18,24]$. However, the level-2.5 LDP contains much more information about the dynamics than the tilted Lindbladian. As well as fluctuations in the quantum jump rates, it also describes fluctuations of the empirical measure $\mu_{\tau}$. Furthermore, it provides the variational principle Eq. (7). These open the door to a range of new studies. We discuss two such directions below.

Application 1: Fluctuation bounds in quantum reset processes.-Classical level-2.5 LDPs have been used to derive lower bounds on the size of fluctuations of currents and fluxes, relating them to entropy production and dynamical activity-these are called "thermodynamic uncertainty relations" (TURs) [34-38]. We now use the variational formula Eq. (7) to obtain similar bounds for open quantum dynamics.

We restrict our analysis to quantum reset processes, in which each jump operator projects the system into a specific state: $\mathcal{J}_{i}(\psi)=f_{i}(\psi) \varphi_{i}$, where $f_{i}$ is a scalar function, and the pure state $\varphi_{i}$ is independent of $\psi$. In this case, the steady-state distribution $P_{\infty}$ is supported on a set of $m$ deterministic paths. It follows that the statistics of jumps can be described by a classical semi-Markov process [46]-the time between jumps is in general a nonexponential random variable with a distribution that depends on the end point of the previous jump (but not on the previous history of the process). For a system that makes a jump of type $i$ at $t=0$, the probability that its next jump is of type $j$ and occurs at time $t$ is $p_{i j}(t)=\operatorname{Tr}\left(J_{j}^{\dagger} J_{j} e^{-i H_{\text {eff }} t} \varphi_{i} e^{i H_{\text {eff }}^{\dagger} t}\right)$. The marginal probability that this jump is of type $j$ is $R_{i j}=\int_{0}^{\infty} d t p_{i j}(t)$, the average time for such a jump is $\tau_{i j}=R_{i j}^{-1} \int_{0}^{\infty} d t t p_{i j}(t)$, and its variance $\sigma_{i j}^{2}=$ $R_{i j}^{-1} \int_{0}^{\infty} d t\left(t-\tau_{i j}\right)^{2} p_{i j}(t)$.

The statistics of jumps in a quantum reset process are fully determined by the $p_{i j}(t)$. Moreover, following Eq. (6), an auxiliary process can be constructed with an arbitrary distribution $\hat{p}_{i j}(t)$. Given such a process whose mean jump rates are $Q^{i}$, one has from Eq. (7) that

$$
I_{1}(Q) \leq \sum_{i j} Q^{i} \int_{0}^{\infty} d t \hat{p}_{i j}(t) \log \frac{\hat{p}_{i j}(t)}{p_{i j}(t)}
$$

where the explicit relation between $Q$ and $\hat{p}_{i j}(t)$ is given in Ref. [41]. Equation (12) provides a lower bound on the 
probability of the rare values of the $Q^{i}$ (with the optimal process saturating the bound and giving the exact $I_{1}$ ).

The above result can be used to establish a general bound on the variance of the empirical rates, which generalizes the classical TURs, as follows (for details, see Ref. [41]). We choose the $\hat{p}_{i j}$ such that all jump times are rescaled uniformly from those of the typical process, $\hat{\tau}_{i j}=\tau_{i j} / a$, where $a$ is a constant, while the marginal probabilities remain the same, $\hat{R}_{i j}=R_{i j}$. This can be achieved with $\hat{p}_{i j}=v_{i j} p_{i j}(t) e^{-u_{i j} t}$ by an appropriate choice of $v_{i j}$ and $u_{i j}$. The jump counts are also rescaled uniformly, $Q^{i}=a \bar{Q}^{i}$, where $\bar{Q}^{i}=\mathbb{E}\left[Q^{i}\right]$ are those of the process $p_{i j}(t)$. Taking $a=1+\delta$ with $\delta \ll 1$, Eq. (12) gives

$$
I_{1}[(1+\delta) \bar{Q}] \leq \frac{1}{2} \chi \delta^{2}+O\left(\delta^{3}\right)
$$

with $\chi=\sum_{i j} \bar{Q}^{i} R_{i j} \tau_{i j}^{2} / \sigma_{i j}^{2}$. This result provides an uncertainty bound for any linear combination of the empirical jump rates, $\mathcal{Q}_{b}=\sum_{i} b_{i} Q^{i}$. That is,

$$
\frac{\operatorname{var}\left(\mathcal{Q}_{b}\right)}{\overline{\mathcal{Q}}_{b}^{2}} \geq \frac{1}{\tau \chi}
$$

where $\overline{\mathcal{Q}}_{b}=\mathbb{E}\left[\mathcal{Q}_{b}\right]=\sum_{i} b_{i} \bar{Q}^{i}$. Equation (14) is a bound on the precision with which $\mathcal{Q}_{b}$ can be estimated, and is thus a TUR for quantum reset processes. In the case where the jump probabilities $p_{i j}(t)$ are exponential-corresponding to a classical jump process-Eq. (14) reduces to the existing classical TUR for counting observables [37], as $\tau_{i j}=\sigma_{i j}$, giving $\chi=\sum_{i j} \bar{Q}^{i} R_{i j}$, which is the average activity. In the open quantum case one may achieve more precise estimates because the bound on precision depends on the reweighed sum in $\chi$. When $\sigma_{i j}<\tau_{i j}$, that is subPoissonian, the more precise jump times can lead to less uncertainty in Eq. (14). Similar enhancement in precision can occur, e.g., in classical systems with time-periodic dynamics [47] or in the presence of magnetic fields [48]. In our case it is related to the possibility of antibunching of quantum jumps [1].

To illustrate the quantum TUR we consider a simple system: a single particle hopping, both coherently and incoherently, between two sites; see Fig. 1(a). Coherent hopping is due to the Hamiltonian $H=$ $\Omega(|10\rangle\langle 01|+| 01\rangle\langle 10|)$. Dissipative incoherent hopping is due to jump operators $J_{L}=\sqrt{\gamma}|10\rangle\langle 01|$ and $J_{R}=$ $\sqrt{\gamma}|01\rangle\langle 10|$. This is a quantum reset process of the kind described above, with reset states $\varphi_{L}=|10\rangle\langle 10|$ and $\varphi_{R}=|01\rangle\langle 01|$. This model, and reset processes in general, can describe experiments, e.g., with quantum dots or superconducting qubits, of topical interest in quantum nonequilibrium and quantum thermodynamics [49-54]. As observable $\mathcal{Q}_{b}$, we consider the flux due to jumps into $\varphi_{L}$, so $\mathcal{Q}_{b}=Q^{L}$. Its rate function $I_{1}\left(Q^{L}\right)$ can be computed (a)
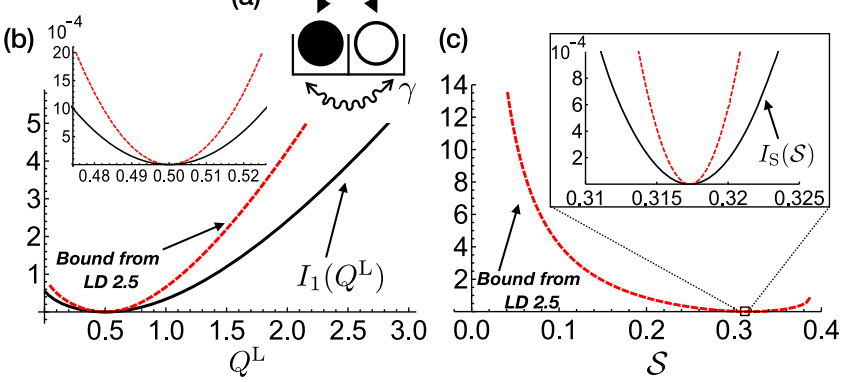

FIG. 1. (a) Example of a quantum reset process: single particle subject to coherent and dissipative hopping. Here, $\Omega=1 / 2$ and $\gamma=1$. (b) Rate function $I_{1}$ for the flux $Q^{L}$ (full black curve) and bound from Eq. (12) (dashed red curve). Inset: Behavior close to the mean corresponding to the TUR Eq. (13). (c) An estimate (upper bound) of the entanglement rate function $I_{S}(\mathcal{S}$ ) (dashed red curve), from Eq. (7). Inset: The bound is compared with the exact $I_{S}(\mathcal{S})$ (full black curve) in the quadratic regime analogous to Eq. $(13) . I_{S}(\mathcal{S})$ is not shown for all $\mathcal{S}$, as its computation requires nontrivial minimization of Eq. (7). However, bounds and estimates can be obtained from simple Ansätze. The largest possible value for $\mathcal{S}_{\tau}$ is $\approx 0.3863$, achieved by long trajectories without incoherent jumps.

from the tilted Lindbladian [18]. Using the Ansatz $\hat{p}_{i j}(t) \propto e^{-u t} p_{i j}(t)$, Eq. (12) yields a bound on the whole rate function, as shown in Fig. 1(b). The inset of Fig. 1(b) shows instead the TUR bound Eq. (13) (dashed red curve) close to the minimum of $I_{1}$ (full black curve).

Application 2: Statistics of time-integrated entanglement.-The level-2.5 LDP Eq. (5) describes the joint fluctuations of $\left(\mu_{\tau}, q_{\tau}\right)$-we have concentrated so far on its implications for the statistics of empirical jump rates. However, the extra information in Eq. (5) may also be exploited to obtain the statistics of nonlinear functions of the state. A prominent example is the entanglement entropy [55].

Consider a bipartite system, where $\psi_{t}$ is the (pure) state of the whole system at time $t$. The entanglement entropy $S_{E}$ between parts $A$ and $B$ is $S_{E}\left(\psi_{t}\right)=-\operatorname{Tr}_{A} \omega\left(\psi_{t}\right) \log \omega\left(\psi_{t}\right)$, where $\omega\left(\psi_{t}\right)=\operatorname{Tr}_{B} \psi_{t}$ is the reduced state in partition $A$, and $\operatorname{Tr}_{A, B}$ denote partial traces over parts $A$ and $B$. In open quantum systems then $\psi_{t}$ is a random (fluctuating) quantity, as is the nonlinear function $S_{E}\left(\psi_{t}\right)$. In particular, the empirical entanglement entropy (i.e., the time average over a trajectory) $\mathcal{S}_{\tau}=\tau^{-1} \int_{0}^{\tau} d t S_{E}\left(\psi_{t}\right)$ obeys a LDP for large time: $\operatorname{Prob}\left[\mathcal{S}_{\tau} \approx \mathcal{S}\right] \simeq \exp \left[-\tau I_{S}(\mathcal{S})\right]$, where $I_{S}$ can again be obtained by contraction from Eq. (5) or by the variational formula Eq. (7), restricted to auxiliary processes where the (mean) entanglement is $\mathcal{S}$. In contrast to the statistics of quantum jumps [18], the fluctuations of $\mathcal{S}_{\tau}$ cannot be obtained by spectral analysis of a tilted Lindbladian. The application of the quantum level-2.5 LDP Eq. (5) to the statistics of nonlinear functions of the state is the third main result of this Letter. 
Figure 1(c) shows the behavior of the empirical entanglement in the example system of Fig. 1(a). In this example, the Lindbladian is unital [2], so the steady state is the identity. As such, the average state has no entanglement for long times. In contrast, the unraveled state $\psi_{t}$ is typically entangled: the evolution with $H_{\text {eff }}=H-i \gamma / 2$ between jumps generates entanglement due to coherent hopping, while jumps return $\psi_{t}$ to the product states $\varphi_{L, R}$, which resets the instantaneous entanglement to zero. The inset in Fig. 1(c) shows the rate function of $S_{\tau}$ (full black curve) as estimated by numerical simulations of the quantum process and the corresponding upper bound (dashed red curve) obtained from Eq. (7), using the same Ansatz as for Fig. 1(b). Computation of the rate function $I_{\mathcal{S}}$ would require exact solution of the variational problem Eq. (7): here we present a bound that applies for all values of $\mathcal{S}$, including the very rare ones (details in Ref. [41]). In general, the exact computation of rate functions will be difficult, but the possibility to bound them with simple Ansätze makes the level-2.5 approach—as in the classical case-both useful and practical.

Outlook.-The level-2.5 method presented here for quantum jump Markov processes can also be formulated for other unravelings, such as those related to homodyne detection experiments and described by diffusive stochastic Belavkin equations [2,3]. Another interesting extension is to discrete time quantum Markovian dynamics. A possible application of such formulation would be to study the statistics of entanglement, out-of-time-order correlators, and operator spreading in random unitary circuits [56-58]. In analogy with classical level-2.5 LDPs, the method here can also be extended to time-periodic dynamics, with possible application to periodically driven (Floquet) quantum systems [59]. We hope to report on some of these extensions in the near future.

This work was supported by EPSRC Grants No. EP/ M014266/1 (J. P. G.) and No. EP/N03404X/1 (F. C. and J.P. G.), and by the European Research Council under the European Union's Seventh Framework Programme (FP/2007-2013)/ERC Grant Agreement No. 335266 (ESCQUMA) (F. C.). We wish to thank Madalin Guta (J. P. G.) and Hugo Touchette (J. P. G. and F. C.) for fruitful discussions.

[1] M. B. Plenio and P. L. Knight, Rev. Mod. Phys. 70, 101 (1998).

[2] H.P. Breuer and F. Petruccione, The Theory of Open Quantum Systems (Oxford University Press, Oxford, 2002).

[3] C. Gardiner and P. Zoller, Quantum Noise (Springer, New York, 2004).

[4] H. M. Wiseman and G. J. Milburn, Quantum Measurement and Control (Cambridge University Press, Cambridge, 2009).

[5] V. Belavkin, Lett. Math. Phys. 20, 85 (1990).

[6] J. Dalibard, Y. Castin, and K. Mølmer, Phys. Rev. Lett. 68, 580 (1992).
[7] C. W. Gardiner, A. S. Parkins, and P. Zoller, Phys. Rev. A 46, 4363 (1992).

[8] H. Carmichael, An Open Systems Approach to Quantum Optics (Springer, Berlin, 1993).

[9] C. Giardina, J. Kurchan, and L. Peliti, Phys. Rev. Lett. 96, 120603 (2006).

[10] J. P. Garrahan, R. L. Jack, V. Lecomte, E. Pitard, K. van Duijvendijk, and F. van Wijland, Phys. Rev. Lett. 98, 195702 (2007).

[11] V. Lecomte, C. Appert-Rolland, and F. van Wijland, J. Stat. Phys. 127, 51 (2007).

[12] J. P. Garrahan, R. L. Jack, V. Lecomte, E. Pitard, K. van Duijvendijk, and F. van Wijland, J. Phys. A 42, 075007 (2009).

[13] H. Touchette, Phys. Rep. 478, 1 (2009).

[14] L. O. Hedges, R. L. Jack, J. P. Garrahan, and D. Chandler, Science 323, 1309 (2009).

[15] R. L. Jack and P. Sollich, Prog. Theor. Phys. Suppl. 184, 304 (2010).

[16] R. Chetrite and H. Touchette, Phys. Rev. Lett. 111, 120601 (2013).

[17] R. Chetrite and H. Touchette, Ann. Henri Poincaré 16, 2005 (2015).

[18] J. P. Garrahan and I. Lesanovsky, Phys. Rev. Lett. 104, 160601 (2010).

[19] C. Ates, B. Olmos, J. P. Garrahan, and I. Lesanovsky, Phys. Rev. A 85, 043620 (2012).

[20] M. Znidaric, Phys. Rev. Lett. 112, 040602 (2014).

[21] M. Znidaric, Phys. Rev. E 89, 042140 (2014).

[22] B. Buca and T. Prosen, Phys. Rev. Lett. 112, 067201 (2014).

[23] F. Carollo, J. P. Garrahan, I. Lesanovsky, and C. PérezEspigares, Phys. Rev. E 96, 052118 (2017).

[24] F. Carollo, J. P. Garrahan, I. Lesanovsky, and C. PérezEspigares, Phys. Rev. A 98, 010103(R) (2018).

[25] J. M. Hickey, S. Genway, I. Lesanovsky, and J. P. Garrahan, Phys. Rev. A 86, 063824 (2012).

[26] C. Maes and K. Netocny, Europhys. Lett. 82, 30003 (2008).

[27] L. Bertini, A. Faggionato, and D. Gabrielli, Stoch. Proc. Appl. 125, 2786 (2015).

[28] A. C. Barato and R. Chetrite, J. Stat. Phys. 160, 1154 (2015).

[29] J. Hoppenau, D. Nickelsen, and A. Engel, New J. Phys. 18, 083010 (2016).

[30] L. Bertini, R. Chetrite, A. Faggionato, and D. Gabrielli, Ann. Henri Poincaré 19, 3197 (2018).

[31] F. den Hollander, S. N. Majumdar, J. M. Meylahn, and H. Touchette, arXiv:1801.09909.

[32] R. Chetrite and H. Touchette, J. Stat. Mech. (2015) P12001.

[33] R. L. Jack and P. Sollich, Eur. Phys. J. Spec. Top. 224, 2351 (2015).

[34] A. C. Barato and U. Seifert, Phys. Rev. Lett. 114, 158101 (2015).

[35] T. R. Gingrich, J. M. Horowitz, N. Perunov, and J. L. England, Phys. Rev. Lett. 116, 120601 (2016).

[36] P. Pietzonka, F. Ritort, and U. Seifert, Phys. Rev. E 96, 012101 (2017)

[37] J. P. Garrahan, Phys. Rev. E 95, 032134 (2017).

[38] A. C. Barato, R. Chetrite, A. Faggionato, and D. Gabrielli, New J. Phys. 20, 103023 (2018).

[39] G. Lindblad, Commun. Math. Phys. 48, 119 (1976). 
[40] V. Gorini, A. Kossakowski, and E. C. G. Sudarshan, J. Math. Phys. (N.Y.) 17, 821 (1976).

[41] See Supplemental Material at http://link.aps.org/ supplemental/10.1103/PhysRevLett.122.130605 for more details on the derivation of the main results.

[42] T. Benoist, M. Fraas, Y. Pautrat, and C. Pellegrini, Probab. Theory Relat. Fields 1 (2018).

[43] Note that the Lindblad equation (1) is a closed equation for the evolution of $\rho=\mathbb{E}[\psi]$. In classical stochastic processes, expectation values do not generally have closed evolution equations. The Belavkin SDE Eq. (2) is special in that it leads to a closed evolution for the average of $\psi_{t}$.

[44] J. P. Garrahan, Physica (Amsterdam) 504A, 130 (2018).

[45] The quantum level-2.5 LDP, Eqs. (10) and (11), contains the one for classical Markov processes as a special case, obtained when $H=0$ with all the $J_{i}$ being rank-1 operators mapping configuration states. In this case, $H_{\text {eff }}$ is diagonal, pure states $\psi$ correspond to individual configurations in the classical basis, and $\mathcal{B}(\psi)$ vanishes. Correspondingly, Eqs. (10) and (11) reduce to the usual classical level-2.5 description.

[46] C. Maes, K. Netočný, and B. Wynants, J. Phys. A 42, 365002 (2009).

[47] A. Barato, R. Chetrite, A. Faggionato, and D. Gabrielli, arXiv:1810.11894.

[48] K. Macieszczak, K. Brandner, and J. P. Garrahan, Phys. Rev. Lett. 121, 130601 (2018).
[49] M. Steffen, M. Ansmann, R. C. Bialczak, N. Katz, E. Lucero, R. McDermott, M. Neeley, E. M. Weig, A. N. Cleland, and J. M. Martinis, Science 313, 1423 (2006).

[50] J. Majer, J. M. Chow, J. M. Gambetta, J. Koch, B. R. Johnson, J. A. Schreier, L. Frunzio, D. I. Schuster, A. A. Houck, A. Wallraff, A. Blais, M. H. Devoret, S. M. Girvin, and R. J. Schoelkopf, Nature (London) 449, 443 (2007).

[51] W. E. Shanks, D. L. Underwood, and A. A. Houck, Nat. Commun. 4, 1991 (2013).

[52] F. W. J. Hekking and J. P. Pekola, Phys. Rev. Lett. 111, 093602 (2013).

[53] D. Husmann, S. Uchino, S. Krinner, M. Lebrat, T. Giamarchi, T. Esslinger, and J.-P. Brantut, Science 350, 1498 (2015).

[54] K. Sachtleben, K. T. Mazon, and L. G. C. Rego, Phys. Rev. Lett. 119, 090601 (2017).

[55] R. Horodecki, P. Horodecki, M. Horodecki, and K. Horodecki, Rev. Mod. Phys. 81, 865 (2009).

[56] A. Nahum, J. Ruhman, S. Vijay, and J. Haah, Phys. Rev. X 7, 031016 (2017).

[57] A. Nahum, S. Vijay, and J. Haah, Phys. Rev. X 8, 021014 (2018).

[58] C. W. von Keyserlingk, T. Rakovszky, F. Pollmann, and S. L. Sondhi, Phys. Rev. X 8, 021013 (2018).

[59] R. Moessner and S. L. Sondhi, Nat. Phys. 13, 424 (2017). 